\title{
Relay-Assisted Multiuser Video Streaming in Cognitive Radio Networks
}

\author{
Yi Xu, Student Member, IEEE, Donglin Hu, and Shiwen Mao, Senior Member, IEEE
}

\begin{abstract}
Due to the drastic increase in wireless video traffic, the capacity of the existing and future wireless networks will be greatly stressed, while interference will become the dominant capacity-limiting factor. In this paper, we investigate relayassisted downlink multiuser video streaming in a cognitive radio (CR) cellular network. We incorporate zero-forcing precoding to allow transmitters collaboratively send encoded (mixed) signals to all CR users, such that undesired signals will be canceled and the desired signal can be decoded at each CR user. We present a stochastic programming formulation of the problem, as well as a problem reformulation that greatly reduces computational complexity. In the cases of a single licensed channel and multiple licensed channels with channel bonding, we develop an optimal distributed algorithm with proven convergence and convergence speed. In the case of multiple channels without channel bonding, we develop a greedy algorithm with a proven performance bound. The algorithms are evaluated with simulations and are shown to achieve considerable gains over two heuristic schemes.
\end{abstract}

Index Terms-Cognitive radio (CR), optimization, relayenhanced cellular network, video streaming, zero forcing.

\section{INTRODUCTION}

D UE TO significant advances in wireless access technologies and the proliferation of wireless devices and applications, there is a fundamental change in wireless network traffic. According to a Cisco study [2], the growth of wireless data is predicted to be 11-fold between 2013 and 2018, and $66 \%$ of the increase in future wireless data will be video related. The capacity of the existing and future wireless networks will be greatly stressed. Coupled with the depleting spectrum and dense chaotic deployment of base stations (BSs), interference will become the major capacity-limiting factor.

Cognitive radios (CRs) provide an effective solution to meet this critical demand, by exploiting codeployed networks and sharing underutilized spectrum [3]. On the other hand, cooperative communications represent another effective solution to the capacity problem, in which wireless nodes

Manuscript received December 30, 2013; revised March 6, 2014; accepted March 22, 2014. Date of publication March 26, 2014; date of current version October 1,2014 . This work was supported in part by the U.S. National Science Foundation under Grants CNS-0953513 and CNS-1247955, and in part by the NSF Broadband Wireless Access and Applications Center, Auburn University, Auburn, AL, USA. Part of this work was done when D. Hu was pursuing a $\mathrm{Ph}$.D. degree at Auburn University. This paper was recommended by Associate Editor J. Liang.

Y. Xu and S. Mao are with the Department of Electrical and Computer Engineering, Auburn University, Auburn, AL 36839-5201 USA (e-mail: yzx0010@tigermail.auburn.edu; smao@ieee.org).

D. Hu is with AT\&T Laboratories, Inc., San Ramon, CA 94583 USA (e-mail: dzh0003@tigermail.auburn.edu).

Color versions of one or more of the figures in this paper are available online at http://ieeexplore.ieee.org.

Digital Object Identifier 10.1109/TCSVT.2014.2313898 help each other in information delivery to achieve the socalled cooperative diversity [4]. Recently, researchers have been exploring the idea of combining these two techniques [5]-[8], and the potential of cooperative CR networks has been demonstrated with a testbed implementation [5]. Furthermore, Guan et al. [9] addressed the challenging problem of joint video encoding rate control, power control, relay selection, and channel in cognitive ad hoc networks with cooperative relays. A solution algorithm based on a combination of the branchand-bound framework and convex relaxation techniques was proposed, and the performance evaluation results demonstrated the efficacy of cooperation and CR for video streaming.

In this paper, we investigate relay-assisted multiuser video streaming in a CR cellular networks, in which videos are supported to make the best use of the enhanced network capacity. We consider a BS and multiple relay nodes (RNs) that collaboratively stream multiple videos to $\mathrm{CR}$ users within the network. It has been shown that the performance of a cooperative relay link is mainly limited by two factors: 1) the half-duplex operation, since the BS-RN and the RN-user transmissions cannot be scheduled simultaneously on the same channel and 2) the bottleneck channel, which is usually the BS-user and/or the RN-user channel, and usually has poor quality due to obstacles, attenuation, multipath propagation, and mobility [4]. To support high-quality video service in such a challenging environment, we assume a well-planned relay network, in which the RNs are connected to the BS with high-speed wireline links. Therefore, the video packets will be available at both the BS and the RNs before their scheduled transmission time, thus allowing advanced cooperative transmission techniques to be adopted for streaming videos.

In particular, we consider zero-forcing precoding, a multiuser interference suppression technique in which the BS and RNs simultaneously transmit encoded mixed signals to all CR users, such that undesired signals will be canceled and the desired signal can be decoded at each CR user [10], [11]. We first present a stochastic programming formulation of the problem of zero forcing for video streaming in cooperative CR networks. The cross-layer optimization formulation considers important design factors including spectrum sensing, opportunistic spectrum access, cooperative relay, zero-forcing precoding, and video quality of service requirements. We then present a reformulation of the problem based on linear algebra theory [12], such that the computational complexity can be greatly reduced.

We develop effective solution algorithms to the formulated problem. In particular, we consider three scenarios. In the case of a single licensed channel, we develop a distributed 
algorithm based on dual decomposition [13] and prove its guaranteed convergence and bounded convergence rate. In the case of multiple licensed channels with channel bonding (in which a transmitter can aggregate all the available channels to transmit the encoded signal [14]), we show that the distributed algorithm can still be used to achieve optimal solutions. Finally, in the case of multiple licensed channels without channel bonding, we develop a greedy algorithm that leverages the single-channel algorithm for near-optimal solutions and prove a lower bound for its performance. The proposed algorithms are evaluated with simulations, and are shown to outperform two heuristic schemes that do not incorporate interference suppression techniques with considerable gains.

The remainder of this paper is organized as follows. We present the system model and preliminaries in Section II and the problem statement in Section III. The proposed solution algorithms are developed and analyzed in Section IV. We present the simulation results in Section $\mathrm{V}$ and discuss related work in Section VI. Section VII concludes this paper.

\section{System Model And PRELIMinaries}

We next present the system model, assumptions, and preliminaries that provide the basis for the problem formulation in Section III. Some of the preliminaries are derived/used in [15] and [16]. We do not claim contribution here but include the preliminaries for the sake of completeness.

\section{A. Spectrum and Network Model}

We consider a spectrum consisting of one common control channel (indexed 0) and $M$ licensed channels (indexed from 1 to $M$ ). The $M$ licensed channels are allocated to a primary network, and the common control channel is exclusively used by a cooperative $\mathrm{CR}$ network colocated with the primary network. As in [3], we assume a synchronized time slot structure for the licensed channels. The states of the $M$ licensed channels evolve over time independently, while the occupancy of each channel follows a two-state discrete time Markov process [3]. Let $\vec{S}(t)=\left[S_{1}(t), S_{2}(t), \ldots, S_{M}(t)\right]$ denote the network state in time slot $t$, where element $S_{m}(t)$ represents the status of channel $m$ as: $S_{m}(t)=0$ for an idle channel and $S_{m}(t)=1$ for a busy channel. The utilization of channel $m$ can be derived as $\eta_{m}=\operatorname{Pr}\left\{S_{m}=1\right\}=\lim _{t_{0} \rightarrow \infty} 1 / t_{0} \sum_{t=1}^{t_{0}} S_{m}(t)$, for $m=1, \ldots, M$.

In the relay-assisted CR cellular network, there is a CR BS (indexed 1) and $(K-1) \mathrm{CR}$ RNs (indexed from 2 to $K$ ) deployed in the area to serve $N$ active CR users. Let $\mathcal{U}=\{1,2, \ldots, N\}$ denote the set of active CR users. We assume that the BS and all the RNs are equipped with multiple transceivers: one is tuned to the common control channel and the others are used to sense multiple licensed channels at the beginning of each time slot, and to transmit encoded signals to $\mathrm{CR}$ users. We consider the case in which each $\mathrm{CR}$ user has one software defined radio (SDR)-based transceiver, which can be tuned to operate on any of the $(M+1)$ channels. If the channel bonding/aggregation techniques are used [14], a transmitter can collectively use all the available channels, and a CR user can receive from all the available channels simultaneously. Otherwise, only one licensed channel will be used by a transmitter, and a CR user can only receive from a single chosen channel at a time.

To support high-quality video service, we assume a wellplanned relay network, in which the RNs are connected to the BS via broadband wireline connections. Although the relays in local thermal equilibrium (LTE)-advanced networks are based on radio interfaces, there are also many solutions based on wireline connections. For example, in small cell networks, the small cell BSs are connected with the X2 interface through wireline connections. In [17], passive optical networks are deployed for the small cell backhaul. In femtocell networks, the femtocell BSs are connected with the broadband wireline connections [16]. In the future cloud radio access network architecture [18], light loaded BSs are connected to a virtualized baseband unit pool through optical connections, in which baseband processing is shared among all the sites. As a result, the video packets will always be available for transmission at the RNs at their scheduled transmission time. To cope with the much poorer BS-user and RN-user channels, the BS and RNs adopt zero forcing to cooperatively transmit multiple videos to $\mathrm{CR}$ users, while exploiting the spectrum opportunities in the licensed channels (Section III).

\section{B. Spectrum Sensing}

Each time slot consists of a spectrum sensing phase and a data transmission phase. The BS and the RNs sense the licensed channels and exchange their sensing results over the common control channel during the sensing phase. We adopt a hypothesis test to detect channel availability. For channel $m$, the null hypothesis and the alternative hypothesis are

$\mathbb{H}_{0}^{m}:\{$ channel $m$ is idle $\}$ and $\mathbb{H}_{1}^{m}:\{$ channel $m$ is busy $\}$.

We model both types of detection errors: 1) false alarm, when an idle channel is considered busy and a spectrum opportunity will be wasted and 2) miss detection, when a busy channel is considered idle, which may cause collision with primary users.

Let $\Theta_{l}^{m}$ be the $l$ th sensing result obtained on channel $m$, with binary ( 0 or 1$)$ values. The false alarm and miss detection probabilities associated with $\Theta_{l}^{m}$, denoted by $\epsilon_{l}^{m}$ and $\delta_{l}^{m}$, are

$$
\epsilon_{l}^{m}=\operatorname{Pr}\left(\Theta_{l}^{m}=1 \mid \mathbb{H}_{0}^{m}\right) \text { and } \delta_{l}^{m}=\operatorname{Pr}\left(\Theta_{l}^{m}=0 \mid \mathbb{H}_{1}^{m}\right) .
$$

Given $L$ sensing results obtained for channel $m$ for time slot $t$, the corresponding sensing result vector is $\vec{\Theta}_{L}^{m}=$ $\left[\Theta_{1}^{m}, \Theta_{2}^{m}, \ldots, \Theta_{L}^{m}\right]$. Let $P_{m}^{A}\left(\vec{\Theta}_{L}^{m}\right):=P_{m}^{A}\left(\Theta_{1}^{m}, \Theta_{2}^{m}, \ldots, \Theta_{L}^{m}\right)$ be the conditional probability that channel $m$ is available, which can be computed iteratively as

$$
\begin{aligned}
P_{m}^{A}\left(\Theta_{1}^{m}\right)= & {\left[1+\frac{\eta_{m}}{1-\eta_{m}} \times \frac{\left(\delta_{1}^{m}\right)^{1-\Theta_{1}^{m}}\left(1-\delta_{1}^{m}\right)^{\Theta_{1}^{m}}}{\left(\epsilon_{1}^{m} \Theta^{\Theta_{1}^{m}}\left(1-\epsilon_{1}^{m}\right)^{1-\Theta_{1}^{m}}\right.}\right]^{-1} } \\
P_{m}^{A}\left(\vec{\Theta}_{l}^{m}\right):= & P_{m}^{A}\left(\Theta_{1}^{m}, \Theta_{2}^{m}, \ldots, \Theta_{l}^{m}\right) \\
= & \left\{1+\left[\frac{1}{P_{m}^{A}\left(\Theta_{1}^{m}, \Theta_{2}^{m}, l, \Theta_{l-1}^{m}\right)}-1\right]\right. \\
& \left.\times \frac{\left(\delta_{l}^{m}\right)^{1-\Theta_{l}^{m}}\left(1-\delta_{l}^{m}\right)^{\Theta_{l}^{m}}}{\left(\epsilon_{l}^{m}\right)^{\Theta_{l}^{m}}\left(1-\epsilon_{l}^{m}\right)^{1-\Theta_{l}^{m}}}\right\}, l=2, \ldots, L .
\end{aligned}
$$


In [19], we show that the more sensing results, the more accurate the channel state estimation. Note that the proposed scheme does not distinguish where the sensing result was obtained. Thus, it is also possible to have the CR users to participate in the sensing phase to collect more sensing results for better prediction of the channel states.

\section{Opportunistic Spectrum Access}

With sensing result $P_{m}^{A}\left(\vec{\Theta}_{L}^{m}\right)$, each channel $m$ will be opportunistically accessed with probability $P_{m}^{D}\left(\vec{\Theta}_{L}^{m}\right)$ in time slot $t$. When channel $m$ is busy (i.e., with probability $1-P_{m}^{A}\left(\vec{\Theta}_{L}^{m}\right)$ ), accessing the channel will cause collision to primary users. For primary user protection, such collision probability should be bounded by a prescribed threshold, denoted as $\gamma_{m}$. The primary user protection constraint can be written as

$$
\left[1-P_{m}^{A}\left(\vec{\Theta}_{L}^{m}\right)\right] P_{m}^{D}\left(\vec{\Theta}_{L}^{m}\right) \leq \gamma_{m} .
$$

Solving (2), the optimal channel access probability is

$$
P_{m}^{D}\left(\vec{\Theta}_{L}^{m}\right)=\min \left\{\gamma_{m} /\left[1-P_{m}^{A}\left(\vec{\Theta}_{L}^{m}\right)\right], 1\right\} .
$$

Define index variables $D_{m}(t)$ 's to indicate the available channels that the BS or RNs access in time slot $t$

$$
D_{m}(t)= \begin{cases}0, & \text { channel } m \text { is accessed in time slot } t \\ 1, & \text { otherwise } \\ & m=1,2, \ldots, M .\end{cases}
$$

Let $\mathcal{A}(t)$ be the set of available channels in time slot $t$. It follows that $\mathcal{A}(t):=\left\{m \mid D_{m}(t)=0\right\}$.

\section{Zero-Forcing Precoding}

We next briefly describe the main idea of zero forcing considered in this paper [10]. Interested readers are referred to [20] and [21] for insightful examples, a classification of various interference alignment scenarios, and practical considerations.

Consider two transmitters (denoted as $s_{1}$ and $s_{2}$ ) and two receivers (denoted as $d_{1}$ and $d_{2}$ ). Let $X_{1}$ and $X_{2}$ be the signals corresponding to the packets to be sent to $d_{1}$ and $d_{2}$, respectively. The transmitters $s_{1}$ and $s_{2}$ send compound signals $a_{1,1} X_{1}+a_{1,2} X_{2}$ and $a_{2,1} X_{1}+a_{2,2} X_{2}$, respectively, to the two receivers $d_{1}$ and $d_{2}$ simultaneously. Let $G_{i, j}$ be the channel gain from transmitter $s_{i}$ to receiver $d_{j}$. If noise is ignored, the received signals $Y_{1}$ and $Y_{2}$ can be written as

$$
\begin{aligned}
{\left[\begin{array}{l}
Y_{1} \\
Y_{2}
\end{array}\right] } & =\left[\begin{array}{ll}
G_{1,1} & G_{1,2} \\
G_{2,1} & G_{2,2}
\end{array}\right]^{\top}\left[\begin{array}{ll}
a_{1,1} & a_{1,2} \\
a_{2,1} & a_{2,2}
\end{array}\right]\left[\begin{array}{l}
X_{1} \\
X_{2}
\end{array}\right] \\
& :=\mathbf{G}^{\top} \times \mathbf{A} \times \vec{X} .
\end{aligned}
$$

From (5), it can be seen that both receivers can perfectly decode their signals if the transformation matrix $\mathbf{A}$ is chosen to be $\left\{\mathbf{G}^{\top}\right\}^{-1}$, i.e., the inverse of the channel gain matrix. With this technique, the transmitters are able to send packets simultaneously, and the interference between the two concurrent transmissions can be effectively canceled at both receivers [21].
For the downlink video streaming application considered in this paper, only the CSI of the downlink channel between a $\mathrm{BS}$ and an RN to the user is required. Therefore, the operation is similar to the traditional cellular networks, in which CSI is also measured for, e.g., power control. The proposed scheme collects CSI in the same fashion as in the existing cellular networks; the only difference may be that the RNs also collect CSI for the channels from themselves to the users. Since relays have been incorporated in modern cellular network standards (e.g., LTE-advanced), it is reasonable to assume that such CSI measurement/feedback is available in practical systems. We assume accurate user channel gains in this paper. The impact of inaccurate measurements is a general problem for adopting interference suppression techniques. Several implementations/testbed experiments have demonstrated the feasibility and robustness of applying interference management techniques in practical settings [20]-[22].

\section{E. Video Performance Measure}

We assume the BS streams multiple real-time videos, one to each active CR user, with help from the RNs. The mediumgrain quality scalability (MGS) option of H.264/Scalable Video Coding (SVC) is adopted in our model because the scalability is very useful to achieve a graceful quality degradation under the highly dynamic CR network environment [15], [23].

Due to the real-time constraint, we assume that each group of pictures (GOPs) must be delivered in the next $T$ time slots. Video packets are transmitted in the decreasing order of their significance to the quality of reconstructed video. To deal with the nonideal channels between BS (RNs) and users, some error control mechanism must be adopted. Due to scalable coding, a lower significance packet will be useless if a higher significance packet is not delivered. As a result, it is necessary to ensure that a higher priority packet is received before sending the next packet. The video will be cut off when the deadline $T$ is reached, and the quality of the reconstructed video is proportional to how many packets can be successfully transmitted within the deadline (with retransmissions and the extra delay incurred). The effect of nonideal channels is captured in the problem formulation.

In [24], a simple linear rate-distortion model is proposed for fine-granular quality scalability (FGS) videos and used in the optimization of FGS video streaming over a wireless local area network (WLAN). Wien et al. [25] provide a representative comparison of FGS and MGS and demonstrate the comparable performance of the two approaches, and the SVC quality scalability videos all exhibit similar linear rate-distortion characteristics for the rate range of interest. Therefore, the quality of reconstructed MGS video can be modeled with a linear equation

$$
W(R)=\alpha+\beta \times R
$$

where $W(R)$ is the average peak signal-to-noise ratio (PSNR) of the reconstructed MGS video, $R$ is the effective received rate of the enhancement layer at the receiver after the channel losses, and $\alpha$ and $\beta$ are constants depending on the specific video sequence and codec. 


\section{Problem Formulation}

We present the problem formulation in this section. As discussed in Section II, the video packets are available at both the BS and all the RNs before their scheduled transmission time; the BS and RNs adopt zero-forcing precoding to overcome the poor BS-CR user and RN-CR user channels.

Let $X_{j}^{m}$ denote the signal to be transmitted to user $j$ on channel $m$, which has unit power. As illustrated in Section II-D, transmitter $k$ sends a compound signal $\sum_{j \in \mathcal{U}} a_{k, j}^{m} X_{j}^{m}$ to all active CR users, where $a_{k, j}^{m}$ 's are the weights to be determined. Ignoring channel noise, we can compute the received signal $Y_{n}^{m}$ at a user $n$ as

$$
\begin{aligned}
Y_{n}^{m} & =\sum_{k=1}^{K} G_{k, n}^{m} \sum_{j=1}^{N} a_{k, j}^{m} X_{j}^{m}=\sum_{k=1}^{K} \sum_{j=1}^{N} a_{k, j}^{m} G_{k, n}^{m} X_{j}^{m} \\
& =\sum_{j=1}^{N} X_{j}^{m} \sum_{k=1}^{K} a_{k, j}^{m} G_{k, n}^{m}, \quad n=1,2, \ldots, N
\end{aligned}
$$

where $G_{k, n}^{m}$ is the channel gain from the BS (i.e., $k=1$ ) or an RN $k$ to user $n$ on channel $m$. Note that to simplify notation, we use $a_{k, j}, G_{k, n}$, and $X_{j}$ instead of $a_{k, j}^{m}, G_{k, n}^{m}$, and $X_{j}^{m}$ when considering only one specific channel. For user $n$, only signal $X_{n}$ should be decoded and the coefficients of all other signals should be forced to zero. The zero-forcing constraints can be written as

$$
\sum_{k=1}^{K} a_{k, j} G_{k, n}=0, \text { for all } j \neq n .
$$

Usually, the total transmit power of the BS and every RN is limited by a peak power $P_{\max }$. Since $X_{j}$ has unit power, for all $j$, the power of each transmitted signal is the square sum of all the coefficients $a_{k, j}^{2}$. The peak power constraint can be written as

$$
\sum_{j=1}^{N}\left|a_{k, j}\right|^{2} \leq P_{\max }, \quad k=1, \ldots, K .
$$

Recall that each CR user has one SDR transceiver that can be tuned to receive from any of the $(M+1)$ channels (without channel bonding). Let $b_{j}^{m}$ be a binary variable indicating that user $j$ selects licensed channel $m$, defined as

$$
b_{j}^{m}= \begin{cases}1, & \text { if user } j \text { receives from channel } m \\ 0, & \text { otherwise } \\ \quad j=1, \ldots, N, m=1, \ldots, M .\end{cases}
$$

Then, we have the following transceiver constraint:

$$
\sum_{m \in \mathcal{A}(t)} b_{j}^{m} \leq 1, \quad j=1, \ldots, N .
$$

Let $w_{j}^{t}$ be the PSNR of user $j$ 's reconstructed video at the beginning of time slot $t$ and $W_{j}^{t}$ the PSNR of user $j$ 's reconstructed video at the end of time slot $t$. In time slot $t$, $w_{j}^{t}$ is already known, while $W_{j}^{t}$ is a random variable depending on the resource allocation and primary user activity during the time slot. That is, $w_{j}^{t+1}$ is a realization of $W_{j}^{t}$.
We formulate a multistage stochastic programming problem to maximize the expected logarithm sum of the PSNRs at the end of the GOP, i.e., $\sum_{j=1}^{N} \mathbb{E}\left[\log \left(W_{j}^{T}\right)\right]$, for proportional fairness among the video sessions. The multistage stochastic programming problem can be decomposed into $T$ serial subproblems, one for each time slot $t$, as [15]

$$
\begin{aligned}
\operatorname{maximize}: & \sum_{j=1}^{N} \mathbb{E}\left[\log \left(W_{j}^{t}\right) \mid w_{j}^{t}\right] \\
\text { s. t } & W_{j}^{t}=w_{j}^{t}+\Psi_{j}^{t} \\
& b_{j}^{m} \in\{0,1\}, \text { for all } m, j
\end{aligned}
$$$$
\text { Constraints (8), (9) and (11) }
$$

where $\Psi_{j}^{t}$ is a random variable depending on spectrum sensing, power allocation, and channel selection in time slot $t$. This is a mixed integer nonlinear programming problem, with binary variables $b_{j}^{m}$ 's and continuous variables $a_{k, j}$ 's.

In particular, $\Psi_{j}^{t}$ can have two possible values: 1) zero, if the packet is not successfully received due to collision with primary users and 2) the objective value increment achieved in time slot $t$ if the packet is successfully received. For the latter case, we have

$$
\begin{aligned}
\Psi_{j}^{t} & =W_{j}(R(t))-W_{j}(R(t-1)) \\
& =\frac{\beta_{j} B}{T} \sum_{m \in \mathcal{A}(t)} \log _{2}\left(1+\frac{1}{N_{0}}\left(\sum_{k=1}^{K} a_{k, j}^{m} G_{k, j}^{m}\right)^{2}\right)
\end{aligned}
$$

where $N_{0}$ is the noise power, $\beta_{j}$ is user $j$ 's constant in the video quality model (6), $B$ is the channel bandwidth, and $T$ is the number of time slots per GOP. To make the problem manageable, we assume the high SINR region, so that $\Psi_{j}^{t}$ in (15) can be approximated as

$$
\Psi_{j}^{t} \approx \frac{\beta_{j} B}{T} \sum_{m \in \mathcal{A}(t)} 2 \log _{2}\left(\frac{1}{\sqrt{N_{0}}}\left(\sum_{k=1}^{K} a_{k, j}^{m} G_{k, j}^{m}\right)\right)
$$

subject to

$$
\sum_{k=1}^{K} a_{k, j}^{m} G_{k, j}^{m}>0 .
$$

Note that (17) corresponds to the data transmitted to user $j$, which has to be greater than zero if user $j$ is active. On the other hand, the zero-forcing constraint (8) ensures that the interference $\sum_{k} a_{k, j} G_{k, n}$ must be zero, when $j \neq n$.

User $j$ can successfully receive a video packet from channel $m$ if it tunes to channel $m$ (i.e., $b_{j}^{m}=1$ ), and the BS and RNs transmit on channel $m$ (i.e., with probability $P_{m}^{D}\left(\vec{\Theta}_{L}^{m}\right)$ ). The probability that user $j$ successfully receives a video packet, denoted as $P_{j}^{t}$, is

$$
P_{j}^{t}=\min \left\{\sum_{m \in \mathcal{A}(t)} b_{j}^{m} P_{m}^{D}\left(\vec{\Theta}_{L}^{m}\right), 1\right\} .
$$


Therefore, we can expand the expectation in (12) to obtain a reformulated problem as follows:

maximize: $\sum_{j=1}^{N} \mathbb{E}\left[P_{j}^{t} \log \left(w_{j}^{t}+\Psi_{j}^{t}\right)+\left(1-P_{j}^{t}\right) \log \left(w_{j}^{t}\right)\right]$

subject to: $\quad$ Constraints (8), (9), (11), (14) and (17).

\section{Solution Algorithms}

In this section, we develop effective solution algorithms to the stochastic programming problem (12). In Section IV-A, we first consider the case of a single licensed channel, and derive a distributed optimal algorithm with guaranteed convergence and bounded convergence speed. We then address the case of multiple licensed channels. If channel bonding/aggregation techniques are used [14], the distributed algorithm in Section IV-A can still be used to achieve optimal solutions. In the case of multiple licensed channels without channel bonding, we develop a greedy algorithm with a performance lower bound in Section IV-C.

\section{A. Case of Single Channel}

1) Property: Consider the case when there is only one licensed channel, i.e., when $M=1$. The $K$ transmitters, including the BS and $(K-1) \mathrm{RNs}$, send video packets to active users using the licensed channel when it is sensed idle.

For user $j$, the weight and channel gain vectors are $\vec{a}_{j}=$ $\left[a_{1, j}, a_{2, j}, \ldots, a_{K, j}\right]^{\top}$ and $\vec{G}_{j}=\left[G_{1, j}, G_{2, j}, \ldots, G_{K, j}\right]^{\top}$, where $T$ denotes matrix transpose. Due to spatial diversity, we assume that the $\vec{G}_{j}$ vectors are linearly independent.

From (8), it can be seen that $\vec{a}_{j}$ is orthogonal to the $(N-1)$ vectors $\vec{G}_{n}$ 's, for $n \neq j$. Since $\vec{a}_{j}$ is a $K$ by 1 vector, there are at most $(K-1)$ vectors that are orthogonal to $\vec{a}_{j}$. Since the $\vec{G}_{j}$ vectors are linearly independent, it follows that $(N-1) \leq$ $(K-1)$ and therefore $N \leq K$. To successfully decode each signal $X_{j}, j=1,2, \ldots, N$, the number of active users $N$ should be smaller than or equal to the number of transmitters $K$.

From (8), it can be seen that $\vec{a}_{j}$ is orthogonal to the $(N-1)$ vectors $\vec{G}_{n}$ 's, for $n \neq j$. Since $\vec{a}_{j}$ is a $K$ by 1 vector, there are at most $(K-1)$ normalized vectors that are orthogonal to $\vec{a}_{j}$. Since the $\vec{G}_{j}$ vectors are linearly independent, it follows that $(N-1) \leq(K-1)$ and therefore $N \leq K$. Therefore, to successfully decode each signal $X_{j}, j=1,2, \ldots, N$, the number of active users $N$ should be smaller than or equal to the number of transmitters $K$.

Note that the above reasoning provides a necessary condition. The following additional constraints should be enforced for the channel selection variables:

$$
\sum_{j=1}^{N} b_{j}^{m} \leq K, \quad \text { for all } m \in \mathcal{A}(t) .
$$

That is, the number of active users receiving from any channel $m$ cannot be more than the number of transmitters on that channel, which is $K$ in the single-channel case and less than or equal to $K$ in the multiple channel case. We first assume that $N$ is not greater than $K$, and will remove this assumption in the following section.

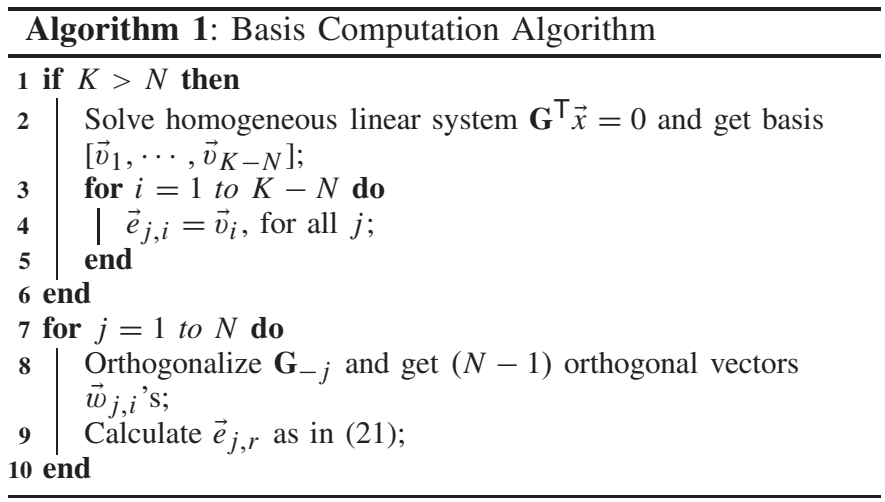

2) Reformulation and Complexity Reduction: With a single channel, all active users receive from channel 1 . Therefore, $b_{j}^{1}=1$, and $b_{j}^{m}=0$, for $m>1, j=1,2, \ldots, N$. The formulated problem is now reduced to a nonlinear programming problem with constraints (8), (9), and (14). If the number of active users is $N=1$, the solution is straightforward: all the transmitters send the same signal $X_{1}$ to the single user using their maximum transmit power $P_{\max }$.

In general, the reduced problem can be solved with the dual decomposition technique [13]. This problem has $K \times N$ primal variables (i.e., the $a_{k, j}$ 's), and we need to define $N(N-1)$ dual variables (or Lagrangian multipliers) for constraints (8) and $K$ dual variables for constraints (9). Before presenting the solution algorithm, we first derive a reformulation of the original problem (19) that can greatly reduce the number of primal and dual variables, such that the computational complexity can be reduced.

Lemma 1: Each vector $\vec{a}_{j}=\left[a_{1, j}, a_{2, j}, \ldots, a_{K, j}\right]^{\top}$ can be represented by the linear combination of $r$ nonzero linearly independent vectors, where $r=K-N+1$.

Proof: From (8), each vector $\vec{a}_{j}$ is orthogonal to $\vec{G}_{i}$, where $j \neq i$. Define a reduced matrix $\mathbf{G}_{-j}$ obtained by deleting $\vec{G}_{j}$ from $\mathbf{G}$, i.e., $\mathbf{G}_{-j}=\left[\vec{G}_{1}, \ldots, \vec{G}_{j-1}, \vec{G}_{j+1}, \ldots, \vec{G}_{N}\right]$. Then, $\vec{a}_{j}$ is a solution to the homogeneous linear system $\mathbf{G}_{-j}^{\top} \vec{x}=0$. Since we assume that the $\vec{G}_{i}$ 's are all linearly independent, the columns of $\mathbf{G}_{-j}$ are also linearly independent [12]. Thus, the rank of $\mathbf{G}_{-j}$ is $(N-1)$. The solution belongs to the null space of $\mathbf{G}_{-j}$. The dimension of the null space is $r=K-(N-1)$ according to the rank-nullity theorem [12]. Therefore, each $\vec{a}_{j}$ can be presented by the linear combination of $r$ linearly independent vectors.

Let $\mathbf{E}_{j}=\left\{\vec{e}_{j, 1}, \vec{e}_{j, 2}, \ldots, \vec{e}_{j, r}\right\}$ be a basis for the null space of $\mathbf{G}_{-j}$. There are many methods to obtain the basis, such as Gaussian elimination. In the following, we present an alternative scheme to compute the basis, which, however, has the same asymptotic complexity as Gaussian elimination.

The algorithm for computing a basis is shown in Algorithm 1. In Steps 1-6, we first solve the homogeneous linear system $\mathbf{G}^{\top} \vec{x}=0$ to get a basis $\left[\vec{v}_{1}, \vec{v}_{2}, \ldots, \vec{v}_{K-N}\right]$. Note that if $K$ is equal to $N$, the basis is the empty set $\emptyset$. We then set the $K-N$ basis vectors to be the first $K-N$ vectors in all the bases $\mathbf{E}_{j}, j=1,2, \ldots, N$. In Step 8, 
we orthogonalize each $\mathbf{G}_{-j}$ and obtain $(N-1)$ orthogonal vectors $\vec{\omega}_{j, i}, i=1,2, \ldots, N-1$. Finally, in Step 9, we let the $r$ th vector $\vec{e}_{j, r}$ be orthogonal to all the $\vec{\omega}_{j, i}$ 's by subtracting all the projections on each $\vec{\omega}_{j, i}$ from $\vec{G}_{j}$, as

$$
\vec{e}_{j, r}=\vec{e}_{j, K-N+1}=\vec{G}_{j}-\sum_{i=1}^{N-1} \frac{\vec{G}_{j}^{\top} \vec{\omega}_{j, i}}{\vec{\omega}_{j, i}^{\top} \vec{\omega}_{j, i}} \vec{\omega}_{j, i}
$$

Lemma 2: The solution space constructed by the basis $\left[\vec{v}_{1}, \vec{v}_{2}, \ldots, \vec{v}_{K-N}\right]$ is a subspace of the solution space of $\mathbf{G}_{-j}^{\top} \vec{x}=0$ for all $j$.

Proof: It can be shown that each vector $\vec{v}_{i}$ is a solution of $\mathbf{G}_{-j}^{\top} \vec{x}=0$, for $i=1,2, \ldots, K-N$.

Lemma 3: The vectors $\left[\vec{v}_{1}, \vec{v}_{2}, \ldots, \vec{v}_{K-N}, \vec{e}_{j, r}\right]$ computed in Algorithm 1 are a basis of the null space of $\mathbf{G}_{-j}$.

Proof: Obviously, the $\vec{v}_{i}$ 's are linearly independent. From (21), it is easy to verify that $\vec{e}_{j, r}$ is orthogonal to all the $\vec{\omega}_{j, i}$ 's. Therefore, $\vec{e}_{j, r}$ is also a solution to system $\mathbf{G}_{-j}^{\top} \vec{x}=0$. Since $\vec{G}_{j}$ and $\vec{\omega}_{j, i}$ are orthogonal to all the $\vec{v}_{i}$ 's, and $\vec{e}_{j, r}$ is a linear combination of $\vec{G}_{j}$ and $\vec{\omega}_{j, i}, \vec{e}_{j, r}$ is also orthogonal and linearly independent to all the $\vec{v}_{i}$ 's. The conclusion follows.

Let $\mathbf{E}_{j}=\left[\vec{v}_{1}, \vec{v}_{2}, \ldots, \vec{v}_{K-N}, \vec{e}_{j, r}\right]$ and define coefficients $\vec{c}_{j}=\left[c_{j, 1}, c_{j, 2}, \ldots, c_{j, r}\right]^{\top}$. Then, we can represent $\vec{a}_{j}$ as a linear combination of the basis vectors, i.e., $\vec{a}_{j}=\sum_{l=1}^{r} c_{j, l} \vec{e}_{j, l}=$ $\mathbf{E}_{j} \vec{c}_{j}$. Equation (16) can be rewritten as

$$
\begin{aligned}
\Psi_{j}^{t} & =\frac{\beta_{j} B}{T} \sum_{m \in \mathcal{A}(t)} 2 \log _{2}\left(\frac{1}{\sqrt{N_{0}}}\left(\vec{c}_{j}^{\top} \mathbf{E}_{j}^{\top} \vec{G}_{j}\right)\right) \\
& =\frac{\beta_{j} B}{T} \sum_{m \in \mathcal{A}(t)} 2 \log _{2}\left(\frac{1}{\sqrt{N_{0}}}\left(c_{j, r} \vec{e}_{j, r}^{\top} \vec{G}_{j}\right)\right) .
\end{aligned}
$$

Note that in the above equation, we omit the channel index $m$ for simple notation. The second equality is because the first $K-N$ column vectors in $\mathbf{E}_{j}$ are orthogonal to $\vec{G}_{j}$. The random variable $W_{j}^{t}$ in the objective function now only depends on $c_{j, r}$. The peak power constraint can be revised as

$$
\sum_{j=1}^{N}\left[\mathbf{E}_{j}(k) \vec{c}_{j}\right]^{2} \leq P_{\max }, \quad k=1, \ldots, K
$$

where $\mathbf{E}_{j}(k)$ is the $k$ th row of matrix $\mathbf{E}_{j}$. In addition, the constraint in (17) can be rewritten as

$$
\left(\mathbf{E}_{j} \vec{c}_{j}\right)^{\top} \vec{G}_{j}>0 .
$$

With such a reformulation, the number of primal and dual variables can be greatly reduced. In Table I, we show the numbers of variables in the original problem and the reformulated problem. The number of primary variables is reduced from $K N$ to $(K-N+1) N$, and the number of dual variables is reduced from $N^{2}+K$ to $N+K$. Such reductions result in greatly reduced computational complexity.

3) Distributed Algorithm: The formulated problem can be solved by the BS in a centralized manner. Alternatively, we develop a distributed algorithm for 1) reducing the computation load at the BS; 2) reducing the burden of the BS on collecting channel states for all users to all the relays; and 3) making the system more robust.
TABLE I

COMPARISON OF COMPUTATIONAL COMPLEXITY FOR THE CASE OF Single ChanNel

\begin{tabular}{l|l|l}
\hline & Original Problem & Reformulated Problem \\
\hline Primal Variables & $K N$ & $(K-N+1) N$ \\
Dual Variables & $N^{2}+K$ & $N+K$ \\
\hline
\end{tabular}

For the distributed algorithm, we define nonnegative dual variables $\vec{\mu}=\left[\mu_{1}, \ldots, \mu_{K}, \lambda_{1}, \ldots, \lambda_{N}\right]^{\top}$ for the inequality constraints. The Lagrangian function is

$$
\begin{aligned}
\mathcal{L}(\mathbf{C}, \vec{\mu})= & \sum_{j=1}^{N} \mathbb{E}\left[\log \left(W_{j}^{t}\left(c_{j, r}\right)\right) \mid w_{j}^{t}\right] \\
& +\sum_{k=1}^{K} \mu_{k}\left(P_{\max }-\sum_{j=1}^{N}\left[\mathbf{E}_{j}(k) \vec{c}_{j}\right]^{2}\right) \\
& +\sum_{j=1}^{N} \lambda_{j}\left(\mathbf{E}_{j} \vec{c}_{j}\right)^{\top} \vec{G}_{j} \\
= & \sum_{j=1}^{N} \mathcal{L}_{j}\left(\vec{c}_{j}, \vec{\mu}\right)+P_{\max } \sum_{k=1}^{K} \mu_{k}
\end{aligned}
$$

where $\mathbf{C}$ is a matrix consisting of all column vector $\vec{c}_{j}$ 's and

$$
\begin{aligned}
\mathcal{L}_{j}\left(\vec{c}_{j}, \vec{\mu}\right)= & \mathbb{E}\left[\log \left(W_{j}^{t}\left(c_{j, r}\right)\right) \mid w_{j}^{t}\right]-\sum_{k=1}^{K} \mu_{k}\left[\mathbf{E}_{j}(k) \vec{c}_{j}\right]^{2} \\
& +\lambda_{j}\left(\mathbf{E}_{j} \vec{c}_{j}\right)^{\top} \vec{G}_{j} .
\end{aligned}
$$

The corresponding problem can be decomposed into $N$ subproblems and solved iteratively [13]. In Step $\tau \geq 1$, for given vector $\vec{\mu}(\tau)$, each CR user solves the following subproblem using local information:

$$
\vec{c}_{j}(\tau)=\arg \max \mathcal{L}_{j}\left(\vec{c}_{j}, \vec{\mu}(\tau)\right) .
$$

Obviously, the objective function in (27) is concave. Therefore, there is a unique optimal solution. The CR users then exchange their solutions over the common control channel. To solve the primal problem, we adopt the gradient method [13]

$$
\vec{c}_{j}(\tau+1)=\vec{c}_{j}(\tau)+\phi \nabla \mathcal{L}_{j}\left(\vec{c}_{j}(\tau), \vec{\mu}(\tau)\right)
$$

where $\nabla \mathcal{L}_{j}\left(\vec{c}_{j}(\tau), \vec{\mu}(\tau)\right)$ is the gradient of the primal problem and $\phi$ is a small positive step size.

The master dual problem for a given $\mathbf{C}(\tau)$ is

$$
\min _{\vec{\mu}} q(\vec{\mu})=\sum_{j=1}^{N} \mathcal{L}_{j}\left(\vec{c}_{j}(\tau), \vec{\mu}\right)+P_{\max } \sum_{k=1}^{K} \mu_{k} .
$$

Since the Lagrangian function is differentiable, the subgradient iteration method can be adopted

$$
\vec{\mu}(\tau+1)=[\vec{\mu}(\tau)-\rho(\tau) \vec{g}(\tau)]^{+}
$$

where $\rho(\tau)=q(\vec{\mu}(\tau))-q\left(\vec{\mu}^{*}\right)\|\vec{g}(\tau)\|^{2}$ is a positive step size, $\vec{\mu}^{*}$ is the optimal solution, $\vec{g}(\tau)=\nabla q(\vec{\mu}(\tau))$ is the gradient of the dual problem, and $[\cdot]^{+}$denotes the projection onto the nonnegative axis. Since the optimal solution $\vec{\mu}^{*}$ is 


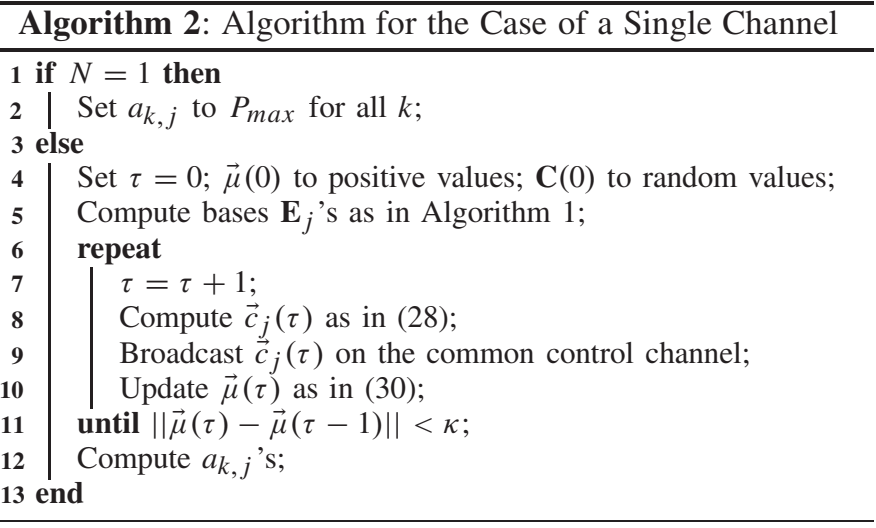

unknown a priori, we choose the mean of the objective values of the primal and dual problems as an estimate for $\vec{\mu}^{*}$ in the algorithm. The updated $\mu_{k}(\tau+1)$ will again be used to solve the subproblems (27). The distributed algorithm is shown in Algorithm 2, where $0 \leq \kappa \ll 1$ is a threshold for convergence.

4) Performance Analysis: We analyze the performance of the distributed algorithm in this section. In particular, we prove that it converges to the optimal solution at a speed faster than $\sqrt{1 / \tau}$ as $\tau$ goes to infinity.

Theorem 1: The series $q(\vec{\mu}(\tau))$ converges to $q\left(\vec{\mu}^{*}\right)$ as $\tau$ goes to infinity and the square error sum $\sum_{\tau=1}^{\infty}(q(\vec{\mu}(\tau))-$ $\left.q\left(\vec{\mu}^{*}\right)\right)^{2}$ is bounded.

Proof: For the optimality gap, we have

$$
\begin{aligned}
\left\|\vec{\mu}(\tau+1)-\vec{\mu}^{*}\right\|^{2}= & \left\|[\vec{\mu}(\tau)-\rho(\tau) \vec{g}(\tau)]^{+}-\vec{\mu}^{*}\right\|^{2} \\
\leq & \left\|\vec{\mu}(\tau)-\rho(\tau) \vec{g}(\tau)-\vec{\mu}^{*}\right\|^{2} \\
= & \left\|\vec{\mu}(\tau)-\vec{\mu}^{*}\right\|^{2} \\
& -2 \rho(\tau)\left(q(\vec{\mu}(\tau))-q\left(\vec{\mu}^{*}\right)\right) \\
& +(\rho(\tau))^{2}\|\vec{g}(\tau)\|^{2} .
\end{aligned}
$$

Since the step size is $\rho(\tau)=q(\vec{\mu}(\tau))-q\left(\vec{\mu}^{*}\right) /\|\vec{g}(\tau)\|^{2}$, it follows that:

$$
\begin{aligned}
\left\|\vec{\mu}(\tau+1)-\vec{\mu}^{*}\right\|^{2} & \leq\left\|\vec{\mu}(\tau)-\vec{\mu}^{*}\right\|^{2}-\frac{\left(q(\vec{\mu}(\tau))-q\left(\vec{\mu}^{*}\right)\right)^{2}}{\|\vec{g}(\tau)\|^{2}} \\
& \leq\left\|\vec{\mu}(\tau)-\vec{\mu}^{*}\right\|^{2}-\frac{\left(q(\vec{\mu}(\tau))-q\left(\vec{\mu}^{*}\right)\right)^{2}}{\hat{g}^{2}}
\end{aligned}
$$

where $\hat{g}^{2}$ is an upper bound of $\|\vec{g}(\tau)\|^{2}$. Since the second term on the right-hand side of (31) is nonnegative, it follows that $\lim _{\tau \rightarrow \infty} q(\vec{\mu}(\tau))=q\left(\vec{\mu}^{*}\right)$.

Summing (31) over $\tau$, we have

$$
\sum_{\tau=1}^{\infty}\left(q(\vec{\mu}(\tau))-q\left(\vec{\mu}^{*}\right)\right)^{2} \leq \hat{g}^{2}\left\|\vec{\mu}(1)-\vec{\mu}^{*}\right\|^{2} .
$$

That is, the square error sum is upper bounded.

Theorem 2: The sequence $\{q(\vec{\mu}(\tau))\}$ converges faster than $\{1 / \sqrt{\tau}\}$ as $\tau$ goes to infinity.

Proof: Assume $\lim _{\tau \rightarrow \infty} \sqrt{\tau}\left(q(\vec{\mu}(\tau))-q\left(\vec{\mu}^{*}\right)\right)>0$. Then, there is a sufficiently large $\tau^{\prime}$ and a positive number $\xi$ such
TABLE II

COMPARISON OF COMPUTATIONAL COMPLEXITY FOR THE CASE OF Multiple Channels With Channel Bonding

\begin{tabular}{l|l|l}
\hline & Original Problem & Reformulated Problem \\
\hline Primal Variables & $K N M$ & $(K-N+1) N M$ \\
Dual Variables & $N^{2} M+K M$ & $N M+K M$ \\
\hline
\end{tabular}

that $\sqrt{\tau}\left(q(\vec{\mu}(\tau))-q\left(\vec{\mu}^{*}\right)\right) \geq \xi$, for all $\tau \geq \tau^{\prime}$. Taking the square sum from $\tau^{\prime}$ to $\infty$, we have

$$
\sum_{\tau=\tau^{\prime}}^{\infty}\left(q(\vec{\mu}(\tau))-q\left(\vec{\mu}^{*}\right)\right)^{2} \geq \xi^{2} \sum_{\tau=\tau^{\prime}}^{\infty} \frac{1}{\tau}=\infty
$$

Equation (32) contradicts with Theorem 1, which states that $\sum_{\tau=1}^{\infty}\left(q(\vec{\mu}(\tau))-q\left(\vec{\mu}^{*}\right)\right)^{2}$ is bounded. Therefore, we have

$$
\lim _{\tau \rightarrow \infty} \frac{q(\vec{\mu}(\tau))-q\left(\vec{\mu}^{*}\right)}{1 / \sqrt{\tau}}=0
$$

indicating that the convergence speed of $q(\vec{\mu}(\tau))$ is faster than that of $1 / \sqrt{\tau}$.

\section{B. Case of Multiple Channels With Channel Bonding}

When there are multiple licensed channels, we first consider the case in which the channel bonding/aggregation techniques are used by the transmitters and CR users [14]). With channel bonding, a transmitter can utilize all the available channels in $\mathcal{A}(t)$ collectively to transmit the mixed signal. We assume that at the end of the sensing phase in each time slot, CR users tune their SDR transceiver to the common control channel to receive the set of available channels $\mathcal{A}(t)$ from the BS. Then, each CR user can receive from all the channels in $\mathcal{A}(t)$ and decode its desired signal from the compound signal it receives.

This case is similar to the case of a single licensed channel. Now, all the active CR users receive from the set of available channels $\mathcal{A}(t)$. We thus have $b_{j}^{m}=1$, for $m \in \mathcal{A}(t)$, and $b_{j}^{m}=0$, for $m \notin \mathcal{A}(t), j=1,2, \ldots, N$. When all the $b_{j}^{m}$ 's are determined this way, (12) is reduced to a nonlinear programming problem with constraints (8) and (9). The distributed algorithm described in Section IV-A can be applied to solve this reduced problem to get optimal solutions. The complexity reduction achieved by the reformulated problem is presented in Table II for this case.

\section{Case of Multiple Channels Without Channel Bonding}

We finally consider the case of multiple channels without channel bonding, in which each CR user has a narrowband SDR transceiver, and can only receive from one of the channels. We first present a greedy algorithm that leverages the optimal algorithm in Algorithm 2 for near-optimal solutions, and then derive a lower bound for its performance. 


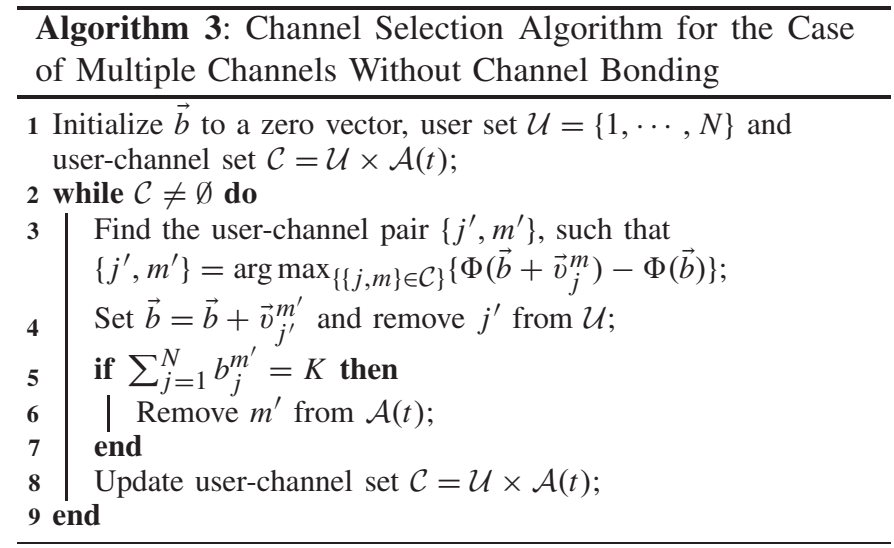

1) Greedy Algorithm: When $M>1$, the optimal solution to (12) depends also on the binary variables $b_{j}^{m}$ 's, which determines whether user $j$ receives from channel $m$. Recall that there are two constraints for the $b_{j}^{m}$ 's: 1) each user can receive from at most one channel (11) and 2) the number of users on the same channel cannot exceed the number of transmitters $K(20)$. Let $\vec{b}$ be the channel allocation vector with elements $b_{j}^{m}$ 's, and $\Phi(\vec{b})$ the corresponding objective value for a given user channel allocation $\vec{b}$.

We take a two-step approach to solve (12). First, we apply the greedy algorithm in Algorithm 3 to choose one available channel in $\mathcal{A}(t)$ for each $\mathrm{CR}$ user (i.e., to determine $\vec{b}$ ). Second, we apply the algorithm in Algorithm 2 to obtain a near-optimal solution for the given channel allocation $\vec{b}$.

In Algorithm 3, $\vec{v}_{j}^{m}$ is a unit vector with 1 for the $[(j-1) \times$ $M+m]$ th element and 0 for all other elements, and $\vec{b}=\vec{b}+\vec{v}_{j^{\prime}}^{m^{\prime}}$ indicates choosing channel $m^{\prime}$ for user $j^{\prime}$. In each iteration, the user-channel pair $\left(j^{\prime}, m^{\prime}\right)$ that can achieve the largest increase in the objective value is chosen, as in Step 3. The worst case complexity of Algorithm 3 is $O\left(K^{2} M^{2}\right)$.

2) Performance Bound: We next analyze the greedy algorithm and derive a lower bound for its performance. Let $v_{l}$ be the sequence from the first to the $l$ th user-channel pair selected by the greedy algorithm. The increase in objective value is denoted as

$$
F_{l}:=F\left(v_{l}, v_{l-1}\right)=\Phi\left(v_{l}\right)-\Phi\left(v_{l-1}\right) .
$$

Sum up (33) from 1 to $S$. We have $\sum_{l=1}^{S} F_{l}=\Phi\left(v_{S}\right)$ since $\Phi\left(v_{0}\right)=0$. Let $\Omega$ be the global optimal solution for user-channel allocation. Define $\pi_{l}$ as a subset of $\Omega$. For given $v_{l}, \pi_{l}$ is the subset of user-channel pairs that cannot be allocated due to the conflict with the $l$ th user channel allocation $v_{l}$ (but not conflict with the user-channel allocations in $\left.v_{l-1}\right)$.

Lemma 4: Assume the greedy algorithm stops in $S$ steps, we have

$$
\Phi(\Omega) \leq \Phi\left(v_{S}\right)+\sum_{l=1}^{S} \sum_{\sigma \in \pi_{l}} F\left(\sigma \cup v_{l-1}, v_{l-1}\right) .
$$

Proof: The proof is similar to the proof of [16, Lemma 7], and is omitted for brevity.

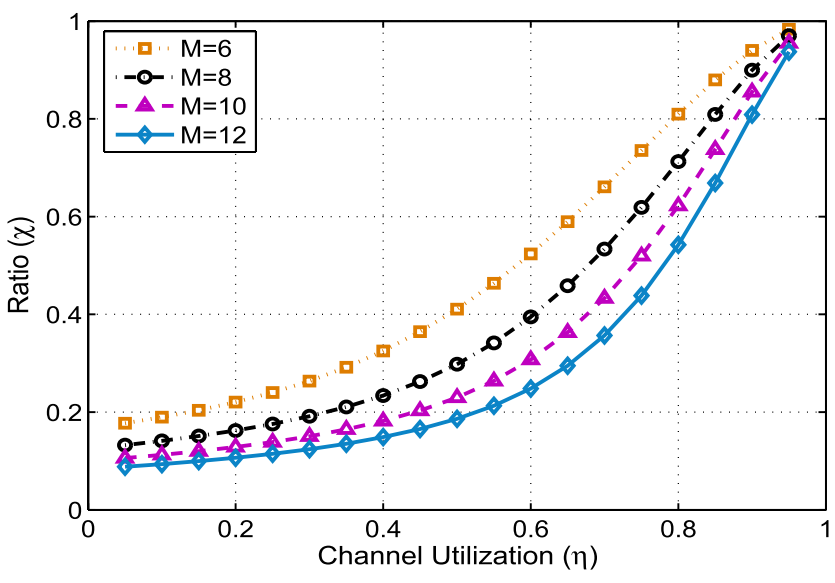

Fig. 1. Competitive ratio $\mathbb{E}[\chi]$ defined in (36) versus channel utilization $\eta$.

Theorem 3: The greedy algorithm for channel selection in Algorithm 3 can achieve an objective value that is at least $1 /|\mathcal{A}(t)|$ of the global optimum in each time slot.

Proof: According to Lemma 4, it follows that:

$$
\begin{aligned}
\Phi(\Omega) & \leq \Phi\left(v_{S}\right)+\sum_{l=1}^{S}\left|\pi_{l}\right| F_{l} \\
& \leq \Phi\left(v_{S}\right)+(|\mathcal{A}(t)|-1) \sum_{l=1}^{S} F_{l} \\
& =|\mathcal{A}(t)| \Phi\left(v_{S}\right) .
\end{aligned}
$$

The second inequality is due to the fact that each user can choose at most one channel, and there are at most $\min \{|\mathcal{A}(t)|-1, N\}$ pairs in $\pi_{l}$ according to the definition. Without loss of generality, we assume there are more secondary users than the number of available channels. Then, the number of pairs in $\pi_{l}$ is at most $(|\mathcal{A}(t)|-1)$. The equality in (34) is because $\sum_{l=1}^{S} F_{l}=\Phi\left(v_{S}\right)$. Then, we have

$$
\frac{1}{|\mathcal{A}(t)|} \Phi(\Omega) \leq \Phi\left(v_{S}\right) \leq \Phi(\Omega) \text {. }
$$

The greedy heuristic solution is lower bounded by $1 /|\mathcal{A}(t)|$ of the global optimum.

Define competitive ratio $\chi=\Phi\left(v_{S}\right) / \Phi(\Omega)=1 /|\mathcal{A}(t)|$. Assume all the licensed channels have identical utilization $\eta$. Define $D$ as the number of available channels. Note that all the licensed channels have identical utilization, and they are identical distributed with Bernoulli distribution. Therefore, $D$ has a binomial distribution as

$$
\operatorname{Pr}(D=d)=\left(\begin{array}{l}
M \\
d
\end{array}\right) \eta^{M-d}(1-\eta)^{d} .
$$

We define $\chi=1$ when there is no available channels. Then, we derive the expectation of $\chi$ as

$$
\mathbb{E}[\chi]=\eta^{M}+\sum_{d=1}^{M}\left(\frac{1}{d}\right)\left(\begin{array}{l}
M \\
d
\end{array}\right) \eta^{M-d}(1-\eta)^{d} .
$$

In Fig. 1, we evaluate the impact of channel utilization $\eta$ and the number of licensed channels $M$ on the competitive ratio. We increase $\eta$ from 0.05 to 0.95 in steps of 0.05 , 
and increase $M$ from 6 to 12 in steps of 2 . The lower bound (35) becomes tighter when $\eta$ is larger, or when $M$ is smaller. For example, when $\eta=0.6$ and $M=6$, the greedy algorithm solution is guaranteed to be no less than $52.7 \%$ of the global optimal. When $\eta$ is increased to 0.95 , the greedy algorithm solution is guaranteed to be no less than $98.3 \%$ of the global optimal. When $|\mathcal{A}(t)|=1$, the proposed scheme is optimal, which validates the earlier analysis in Section IV-A.

\section{Performance Evaluation}

We evaluate the performance of the proposed algorithms with a MATLAB implementation and the JSVM 9.13 video codec. For comparison, we develop two simpler heuristic schemes that do not incorporate interference alignment.

1) Heuristic 1: each $\mathrm{CR}$ user selects the best channel in $\mathcal{A}(t)$ based on channel condition. The time slot is equally divided among the users receiving from the same channel, to send their signals separately in each time slot.

2) Heuristic 2: in each time slot, the active user with the best channel is selected for each available channel. The entire time slot is used to transmit this user's signal.

Once channel assignment is determined, the PSNR increment in time slot $t$ achieved by Heuristic 1 can be derived as

$$
\Psi_{j}^{t}=\frac{\beta_{j} B}{T} \sum_{m \in \mathcal{A}(t)} \frac{b_{j}^{m}}{\sum_{j} b_{j}^{m}} \log _{2}\left(1+\frac{1}{N_{0}} \sum_{k=1}^{K}\left(G_{k, j}^{m}\right)^{2}\right) .
$$

Similarly, $\Psi_{j}^{t}$ achieved by Heuristic 2 can be derived as

$$
\Psi_{j}^{t}=\frac{\beta_{j} B}{T} \sum_{m \in \mathcal{A}(t)} b_{j}^{m} \log _{2}\left(1+\frac{1}{N_{0}} \sum_{k=1}^{K}\left(G_{k, j}^{m}\right)^{2}\right) .
$$

As discussed in Section II-A, the dynamic of the licensed channels is modeled by two-state discrete-time Markov processes. For the user channels, the channel fading-gain process is piecewise constant on blocks of one time slot, and fading in different time slots are independent. The Rayleigh fading model is used for all the simulation results reported in this paper. The proposed algorithm uses the video quality model (6) when solving the formulated problem. Then, the solution is used in the simulations driven by the video data generated by the JSVM 9.13 video codec using different test sequences.

\section{A. Case of Single Licensed Channel}

In the first scenario, there are $K=4$ transmitters, i.e., one BS and three RNs. The channel utilization $\eta$ is set to 0.6 , and the maximum allowable collision probability $\gamma$ is set to 0.2. There are three active CR users, each receives an MGS video stream from the BS: Bus to CR user 1, Mobile to CR user 2, and Harbor to CR user 3. The video sequences are in the common intermediate format $(252 \times 288)$. The GOP size of the videos is 16 , and the delivery deadline $T$ is 10 . The false alarm probability is $\epsilon_{l}^{m}=0.3$ and the miss detection probability is $\delta_{l}^{m}=0.3$ for all spectrum sensors. The channel

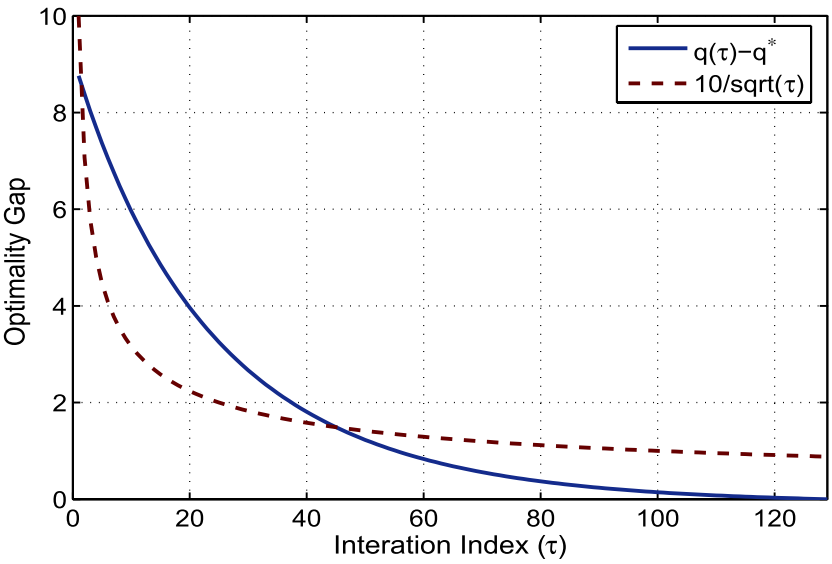

Fig. 2. Convergence rate of the distributed algorithm with a single channel.

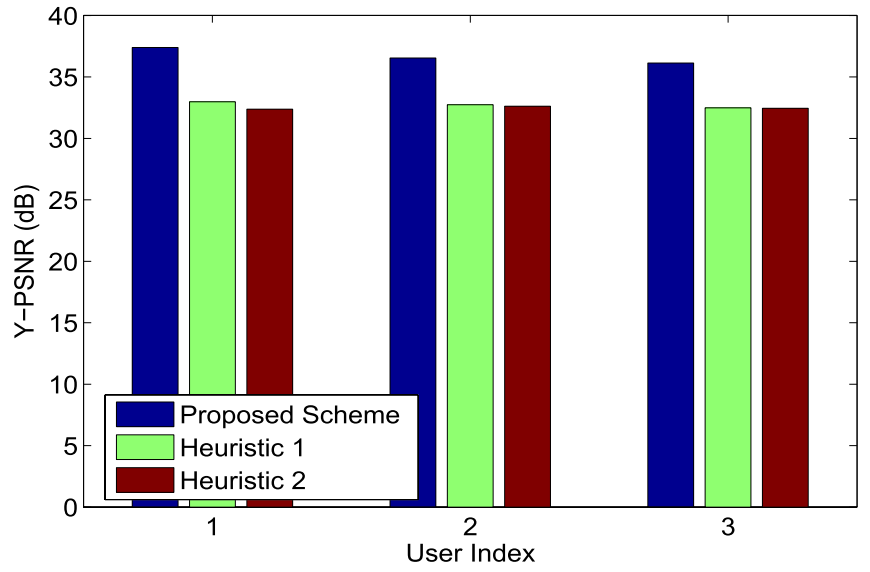

Fig. 3. Received video quality for each CR user with a single channel.

bandwidth $B$ is $1 \mathrm{MHz}$. The peak power limit is $10 \mathrm{~W}$ for all the transmitters, unless otherwise specified.

We first examine the convergence rate of the distributed algorithm. According to Theorem 2, the distributed algorithm converges at a speed faster than $1 / \sqrt{\tau}$ asymptotically. We compare the optimality gap of the proposed algorithm, i.e., $\left|q(\tau)-q^{*}\right|$, with series $10 / \sqrt{\tau}$ in Fig. 2. Both curves converge to zero as $\tau$ goes to infinity. It can be seen that the convergence speed, i.e., the slope of the curve, of the proposed scheme is larger than that of $10 / \sqrt{\tau}$ after about 10 iterations. The convergence of the optimality gap is much faster than $10 / \sqrt{\tau}$, which exhibits a heavy tail.

We next present the average Y-PSNRs of the three reconstructed MGS videos in Fig. 3. Among three schemes, the proposed algorithm achieves the highest PSNR value. To illustrate the visual quality of the reconstructed video, we plotted selected frames for each user in Fig. 4. The first heuristic algorithm has an inferior performance since there is no collaboration among the transmitters, and time-division multiple access has to be adopted to avoid interference among the users choosing the same channel. The second heuristic algorithm has the poorest performance, since for each time slot only one cognitive user is active. Note that the proposed algorithm is optimal in the single-channel case. It achieves significant 


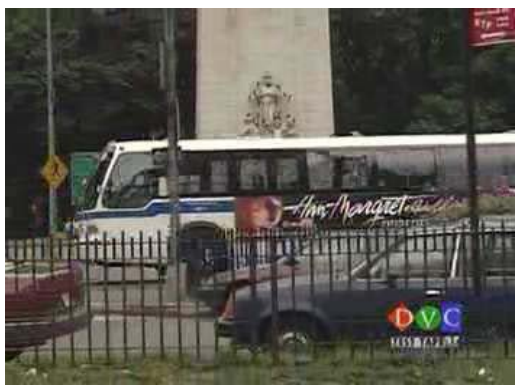

(a)

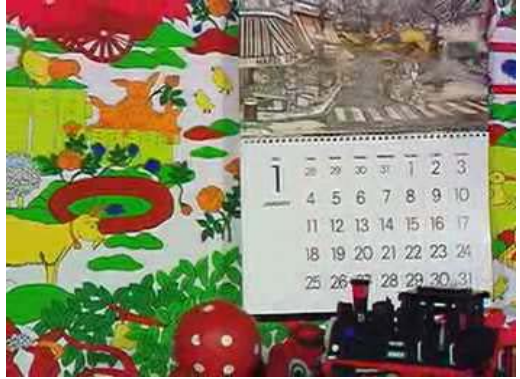

(d)

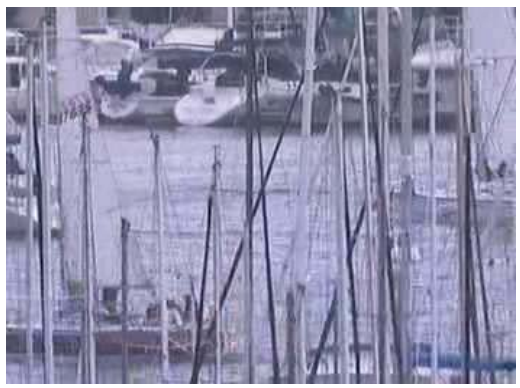

(g)

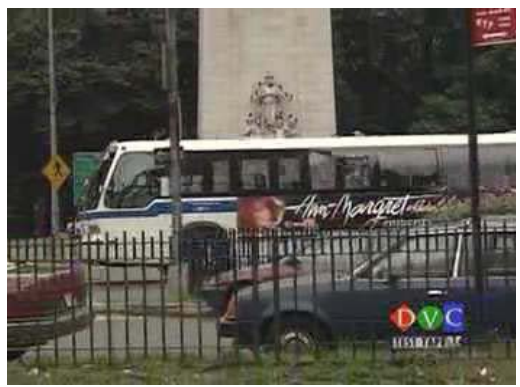

(b)

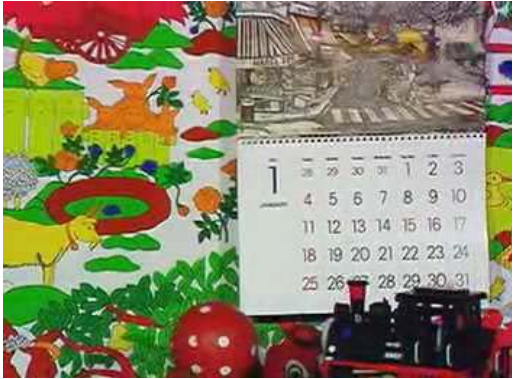

(e)

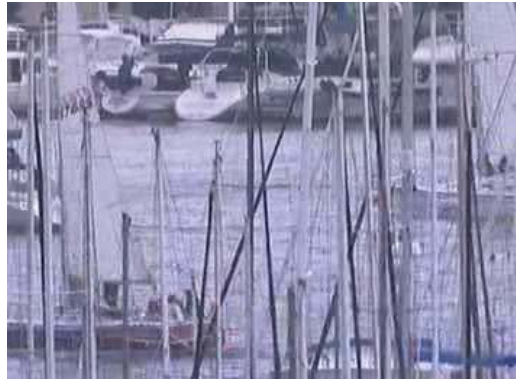

(h)

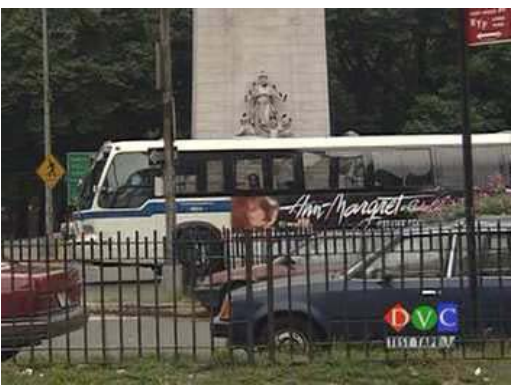

(c)

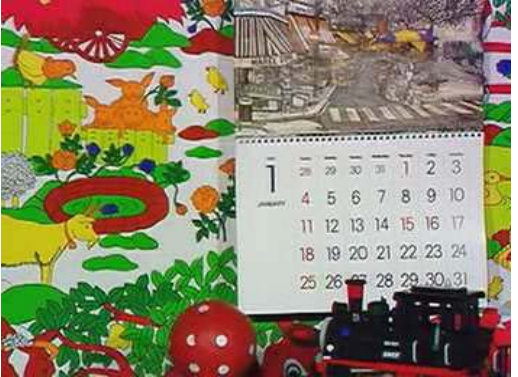

(f)

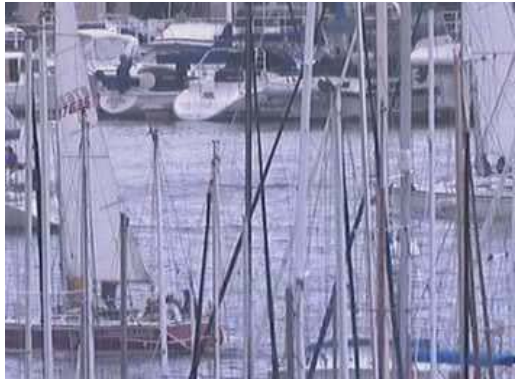

(i)

Fig. 4. Comparison of the visual quality of the reconstructed frames for the three users. (a) User1, Bus, Heuristic1. (b) User1, Bus, Heuristic2. (c) User1, Bus, Proposed. (d) User2, Mobile, Heuristic2. (e) User2, Mobile, Proposed. (f) User3, Harbor, Heuristic1. (g) User3, Harbor, Heuristic2. (h) User3, Harbor, Proposed. (i) User3, Harbor, Proposed.

improvements ranging from 3.64 to $5.02 \mathrm{~dB}$ over the two heuristic algorithms.

\section{B. Case of Multiple Channels With Channel Bonding}

In the second scenario, we investigate the case with two licensed channels with channel bonding. The parameters are the same as in the previous section, except that $M=2$. We also assume that CR users $1-3$ are streaming test sequence Bus, Mobile, and Harbor, respectively. The simulation results are plotted in Figs. 5 and 6. Similar trend can be observed as in Fig. 3, where the superior performance of the proposed scheme is demonstrated.

For the two heuristic algorithms, we find that $\sum_{j} \Psi_{j}^{t}$ in Heuristic 1 is upper bounded by that of Heuristic 2 . However, due to the effect of the initial values of $w_{j}^{t}$, which is essentially $\alpha_{i}$, and the bound of success probability in (18), the average Y-PSNR of Heuristic 1 is not necessarily lower than that of Heuristic 2. For instance, in the single-channel case, the average Y-PSNR of Heuristic 1 is higher than that of Heuristic 2, since in Heuristic 2, only one CR user is selected. As the number of channels is increased to two, two
CR users will be selected with Heuristic 2. Hence, the Y-PSNR performance of Heuristic 2 catches up. We can expect that if the number of available channels is greater than or equal to the number of CR users, Heuristic 2 will achieve much higher Y-PSNR values than Heuristic 1.

Comparing with the proposed scheme, the two heuristic algorithms have the advantage of low complexity. These two schemes also exploit the diversity gain in multiuser communications. These can be adopted when complexity or scalability becomes the main objective than video quality.

\section{Case of Multiple Channels Without Channel Bonding}

We next investigate the third scenario with six licensed channels and four transmitters. There are 12 CR users, each streaming one of the three different videos Bus, Mobile, and Harbor. The rest of the parameters are the same as those in the single-channel case, unless otherwise specified. Equation (34) can also be interpreted as an upper bound on the global optimal, i.e., $\Phi(\Omega) \leq|\mathcal{A}(t)| \Phi\left(v_{L}\right)$, which is also plotted in the figures. Each point in the following figures is the average of 10 simulation runs with different random seeds. 


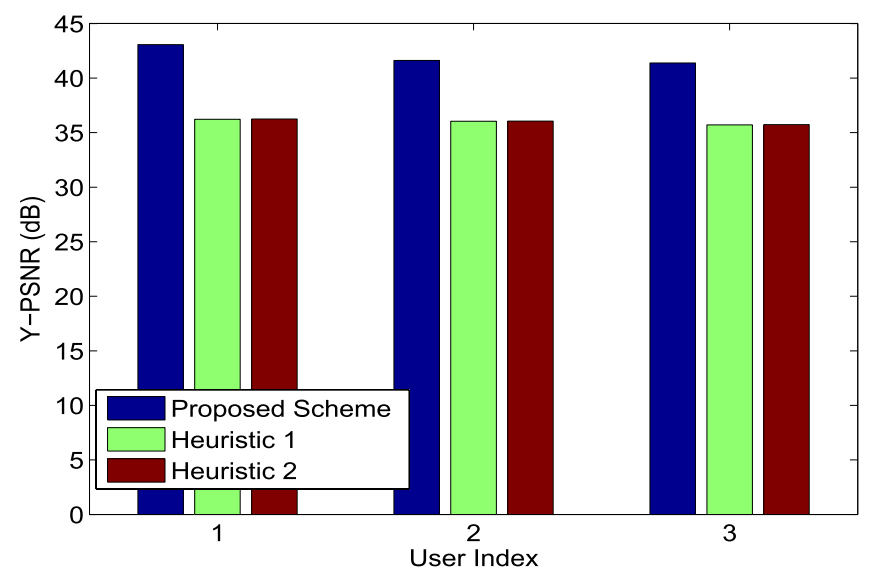

Fig. 5. Received video quality for each CR user for multiple channels with channel bonding.

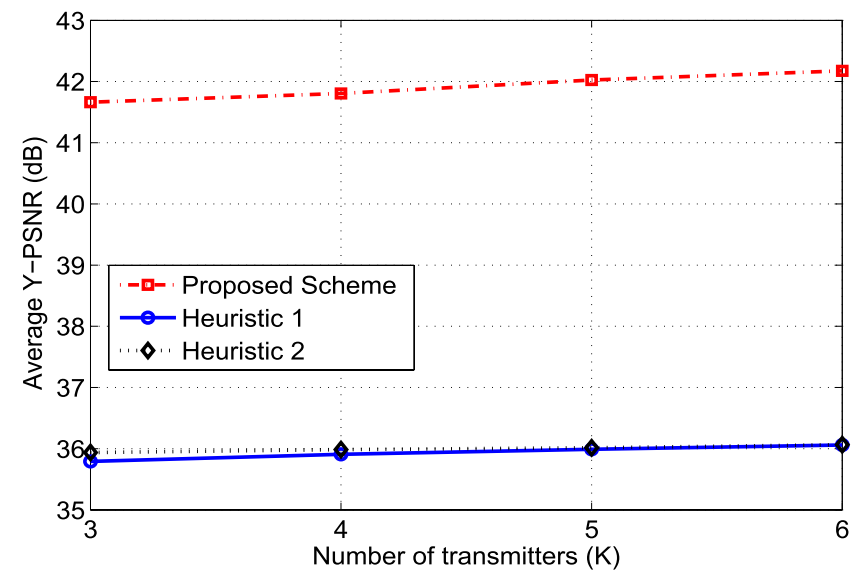

Fig. 6. Reconstructed video quality versus the number of transmitters $K$ for multiple channels with channel bonding.

The $95 \%$ confidence intervals are plotted as error bars, which are generally negligible.

The impact of channel utilization $\eta$ on received video quality is shown in Fig. 7. We increase $\eta$ from 0.3 to 0.9 in steps of 0.15 , and plot the Y-PSNRs of reconstructed videos averaged over all the $12 \mathrm{CR}$ users. Intuitively, a smaller $\eta$ allows more transmission opportunities for CR users, thus allowing the CR users to achieve higher video rates and better video quality. This is shown in the figure, in which all four curves decrease as $\eta$ is increased. We also observe that the gap between the upper bound and proposed schemes becomes smaller as $\eta$ gets larger, from $32.65 \mathrm{~dB}$ when $\eta=0.3$ to $0.63 \mathrm{~dB}$ when $\eta=0.9$. This trend is also shown in Fig. 1 . The proposed scheme outperforms the two heuristic schemes with considerable gains, ranging from 0.8 to $3.65 \mathrm{~dB}$.

Finally, we investigate the impact of the number of transmitters $K$ on the video quality. In this simulation, we increase $K$ from two to six with step size one. The average Y-PSNRs of all the $12 \mathrm{CR}$ users are plotted in Fig. 8. As expected, the more transmitters, the more effective the interference alignment technique, and thus the better the video quality. The proposed algorithm achieves gains ranging from 1.78

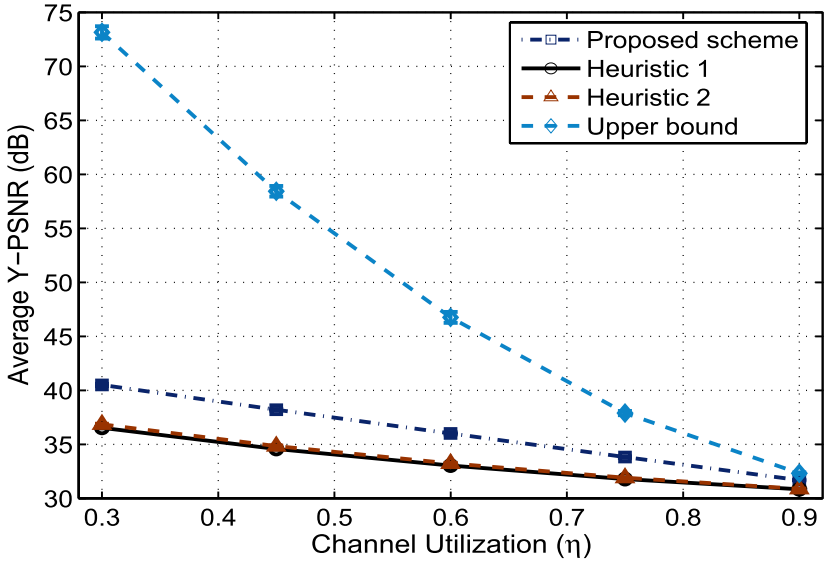

Fig. 7. Reconstructed video quality versus channel utilization $\eta$ in the multichannel without channel bonding case.

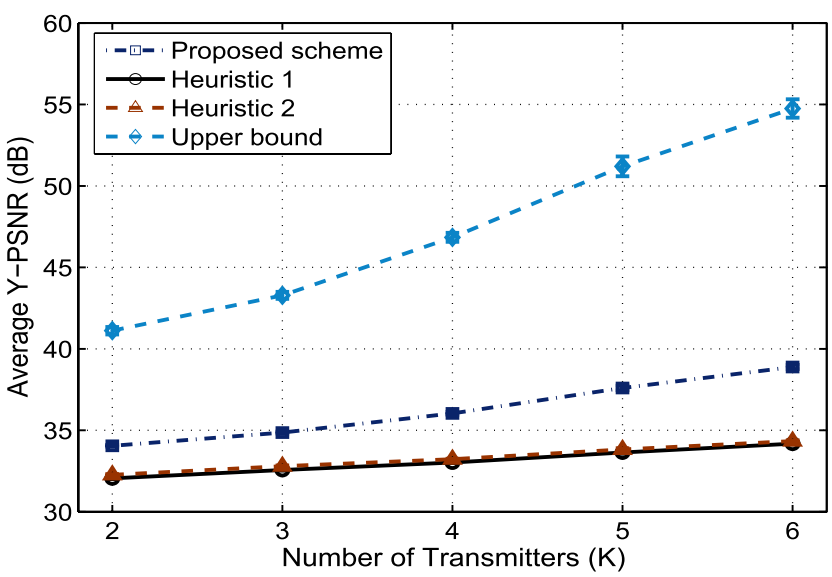

Fig. 8. Reconstructed video quality versus number of transmitters $K$ in the multichannel without channel bonding case.

(when $K=2$ ) to $4.55 \mathrm{~dB}$ (when $K=6$ ) over the two heuristic schemes.

\section{RElated Work}

Video over wireless networks have been an active research area for years [26]-[30]. This paper is closely related to the prior work on cooperative communications [4] and that on CR networking [3]. There have been significant advances in these areas, which laid out the foundation for this paper. Researchers have been exploring the idea of combining these two techniques [5], [6], [8]. In [5], an overview of cooperative relay scenarios and related issues was presented, along with a GNU radio implementation of a Medium Access Control protocol. In [6], a centralized heuristic was presented to address the relay selection and spectrum allocation problem in CR networks.

Cooperative diversity has been exploited for wireless video streaming in [9] and [31]-[34]. Liu et al. [31] proposed a network-coding-based cooperative repair framework for peers in an ad hoc WLAN to improve broadcast video quality during channel losses, in which repair is optimized for broadcast video in a rate-distortion manner. Hua et al. [32] proposed 
a scalable video broadcast/multicast solution that integrates scalable video coding, 3G broadcast, and ad hoc relays to balance the system-wide and worst case video quality of all viewers in a $3 \mathrm{G}$ cell. In [33], two-hop cooperative transmission was integrated with layered video coding and packet-level forward error correction to enable efficient and robust video multicast in a cellular network, in which multiple relays forward the data simultaneously using randomized distributed space time codes. In [34], a problem of joint rate control, relay selection, and power allocation for video streaming over cooperative networks was formulated and solved, aiming to maximize the sum PSNR of a set of concurrent video sessions.

The problem of video over CR networks has only been studied in [15], [16], [23], and [35]-[38]. In [35], a dynamic channel selection scheme was proposed for CR users to transmit videos over multiple channels. In [36], a distributed joint routing and spectrum sharing algorithm for video streaming over CR ad hoc networks was described and evaluated with simulations. In our prior work, we considered video multicast in an infrastructure-based CR network [15], unicast video streaming over multihop CR networks [23], and CR femtocell networks [16]. In [37], the impact of system parameters residing in different network layers is jointly considered to achieve the best possible video quality for CR users. Unlike the heuristic approaches in [35] and [36], the analytical and optimization approach taken in this paper yields algorithms with optimal or bounded performance. Ding and Xiao [38] investigated the problem of enabling multisource video on-demand applications in multiinterface cognitive wireless mesh networks. Both centralized and distributed algorithms are developed for joint multipath routing and spectrum allocation for video sessions to minimize the total bandwidth cost.

As point-to-point link capacity approaches the Shannon limit, there has been considerable interest on exploiting interference to improve wireless network capacity [10], [11], [20], [21], [39]. In addition to information theoretic work on asymptotic capacity [10], [11], practical issues have been addressed in [20]-[22], [39], and [40]. Katti et al. [39] presented a practical design of analog network coding to exploit interference and allow concurrent transmissions, which does not make any synchronization assumptions. In [20], interference alignment and cancellation is incorporated in multiple-input and multiple-output LANs, and the network capacity is shown, analytically and experimentally, to be almost doubled. Li et al. [21] presented a general algorithm for identifying interference alignment and cancellation opportunities in practical multihop mesh networks. The impact of synchronization and channel estimation was evaluated through a GNU radio implementation. Liu et al. [40] present a relayassisted ARQ scheme, in which a distributed beamformingbased interference cancellation scheme is used for cognitive relays to exploit spectrum opportunities for retransmission of lost packets. Anand et al. [22] presented the design and experimental evaluation of Simultaneous Transmission with Orthogonally Blinded Eavesdroppers, in which multiantenna APs can construct simultaneous data streams using zero-forcing beamforming. This work certainly justified the viability of the proposed scheme in this paper.

\section{CONCLUSION}

In this paper, we investigated the problem of relay-assisted multiuser scalable video streaming in a relay enhanced CR cellular network. We presented a stochastic programming formation and derived a reformulation that leads to considerable reduction in computational complexity. A distributed optimal algorithm was developed for the case of a single channel and the case of multichannel with channel bonding, with proven convergence and convergence speed. We also presented a greedy algorithm for the multichannel without channel bonding case, with a proven performance bound. The proposed algorithms were evaluated with simulations and were shown to outperform two heuristic schemes without interference alignment with considerable gains.

\section{REFERENCES}

[1] D. Hu and S. Mao, "Cooperative relay with interference alignment for video over cognitive radio networks," in Proc. IEEE INFOCOM, Orlando, FL, USA, Mar. 2012, pp. 2014-2022.

[2] Cisco. (2014, Feb.). Cisco Visual Networking Index: Global Mobile Data Traffic Forecast Update, 2013-2018 [Online]. Available: http://www.cisco.com/

[3] Q. Zhao and B. Sadler, "A survey of dynamic spectrum access," IEEE Signal Process. Mag., vol. 24, no. 3, pp. 79-89, May 2007.

[4] J. N. Laneman, D. Tse, and G. Wornell, "Cooperative diversity in wireless networks: Efficient protocols and outage behavior," IEEE Trans. Inf. Theory, vol. 50, no. 12, pp. 3062-3080, Dec. 2004.

[5] Q. Zhang, J. Jia, and J. Zhang, "Cooperative relay to improve diversity in cognitive radio networks," IEEE Commun. Mag., vol. 47, no. 2, pp. 111-117, Feb. 2009.

[6] J. Jia, J. Zhang, and Q. Zhang, "Cooperative relay for cognitive radio networks," in Proc. IEEE INFOCOM, Apr. 2009, pp. 2304-2312.

[7] D. $\mathrm{Hu}$ and S. Mao, "Cooperative relay in cognitive radio networks: Decode-and-forward or amplify-and-forward?" in Proc. IEEE GLOBECOM, Miami, FL, USA, Dec. 2010, pp. 1-5.

[8] D. Hu and S. Mao, "On cooperative relay in cognitive radio networks," EAI Endorsed Trans. Mobile Commun. Appl., vol. 1, no. 3, p. e3, Dec. 2013.

[9] Z. Guan, L. Ding, T. Melodia, and D. Yuan, "On the effect of cooperative relaying on the performance of video streaming applications in cognitive radio networks," in Proc. IEEE ICC, Kyoto, Japan, Jun. 2011, pp. 1-6.

[10] Q. Spencer, A. L. Swindlehurst, and M. Haardt, "Zero-forcing methods for downlink spatial multiplexing in multiuser MIMO channels," IEEE Trans. Signal Process., vol. 52, no. 2, pp. 461-471, Feb. 2004.

[11] V. Cadambe and S. A. Jafar, "Interference alignment and the degrees of freedom for the $k$ user interference channel," IEEE Trans. Inf. Theory, vol. 54, no. 8, pp. 3425-3441, May 2008.

[12] G. Strang, Introduction to Linear Algebra, 4th ed. Cambridge, MA, USA: Wellesley-Cambridge, 2009.

[13] D. P. Bertsekas, Nonlinear Programming, 2nd ed. Nashua, NH, USA: Athena Scientific, 1999.

[14] H. Mahmoud, T. Yucek, and H. Arslan, "OFDM for cognitive radio: Merits and challenges," IEEE Wireless Commun. Mag., vol. 16, no. 2, pp. 6-14, Apr. 2009.

[15] D. Hu and S. Mao, "Streaming scalable videos over multi-hop cognitive radio networks," IEEE Trans. Wireless Commun., vol. 9, no. 11, pp. 3501-3511, Nov. 2010.

[16] D. Hu and S. Mao, "On medium grain scalable video streaming over cognitive radio femtocell networks," IEEE J. Sel. Areas Commun., vol. 30, no. 3, pp. 641-651, Apr. 2012.

[17] C. Ranaweera, P. Iannone, K. Oikonomou, K. Reichmann, and R. Sinha, "Design of cost-optimal passive optical networks for small cell backhaul using installed fibers [Invited]," IEEE/OSA J. Opt. Commun. Netw., vol. 5, no. 10, pp. A230-A239, Oct. 2013. 
[18] Y. Lin, L. Shao, Z. Zhu, Q. Wang, and R. K. Sabhikhi, "Wireless network cloud: Architecture and system requirements," IBM J. Res. Develop., vol. 54, no. 1, pp. 4:1-4:12, Jan. 2010.

[19] D. Hu and S. Mao, "A sensing error aware MAC protocol for cognitive radio networks," EAI Endorsed Trans. Mobile Commun. Appl., vol. 12, nos. 7-9, Aug. 2012, p. e1.

[20] S. Gollakota, S. David, and D. Katabi, "Interference alignment and cancellation," in Proc. ACM SIGCOMM, Barcelona, Spain, Aug. 2009, pp. $159-170$.

[21] L. E. Li, R. Alimi, D. Shen, H. Viswanathan, and Y. R. Yang, "A general algorithm for interference alignment and cancellation in wireless networks," in Proc. IEEE INFOCOM, San Diego, CA, USA, Mar. 2010, pp. 1-9.

[22] N. Anand, S. Lee, and E. Knightly, "STROBE: Actively securing wireless communications using zero-forcing beamforming," in Proc. IEEE INFOCOM, Orlando, FL, USA, Mar. 2012, pp. 720-728.

[23] D. Hu, S. Mao, Y. T. Hou, and J. H. Reed, "Fine grained scalability video multicast in cognitive radio networks," IEEE J. Sel. Areas Commun., vol. 28, no. 3, pp. 334-344, Apr. 2010.

[24] M. van der Schaar, S. Krishnamachari, S. Choi, and X. Xu, "Adaptive cross-layer protection strategies for robust scalable video transmission over 802.11 WLANs," IEEE J. Sel. Areas Commun., vol. 21, no. 10, pp. 1752-1763, Dec. 2003.

[25] M. Wien, H. Schwarz, and T. Oelbaum, "Performance analysis of SVC," IEEE Trans. Circuits Syst. Video Technol., vol. 17, no. 9, pp. 1194-1203, Sep. 2007.

[26] Z. Liu, G. Cheung, and Y. Ji, "Optimizing distributed source coding for interactive multiview video streaming over lossy networks," IEEE Trans. Circuits Syst. Video Technol., vol. 23, no. 10, pp. 1781-1794, Oct. 2013

[27] Z. Yang and X. Wang, "Scalable video broadcast over downlink MIMO-OFDM systems," IEEE Trans. Circuits Syst. Video Technol., vol. 23, no. 2, pp. 212-223, Feb. 2013

[28] S. Mao, Y. T. Hou, X. Cheng, H. D. Sherali, S. F. Midkiff, and Y.-Q. Zhang, "On routing for multiple description video over wireless ad hoc networks," IEEE Trans. Multimedia, vol. 8, no. 5, pp. 1063-1074, Oct. 2006

[29] S. Kompella, S. Mao, Y. T. Hou, and H. D. Sherali, "Cross-layer optimized multipath routing for video communications in wireless networks," IEEE J. Sel. Areas Commun., vol. 25, no. 4, pp. 831-840, May 2007.

[30] C. Li, D. Wu, and H. Xiong, "Delay-power-rate-distortion model for wireless video communication under delay and energy constraints," IEEE Trans. Circuits Syst. Video Technol., 2013.

[31] X. Liu, G. Cheung, and C.-N. Chuah, "Structured network coding and cooperative wireless ad-hoc peer-to-peer repair for WWAN video broadcast," IEEE Trans. Multimedia, vol. 11, no. 4, pp. 730-741, Jun. 2009

[32] S. Hua, Y. Guo, Y. Liu, H. Liu, and S. S. Panwar, "Scalable video multicast in hybrid 3G/ad-hoc networks," IEEE Trans. Multimedia, vol. 13, no. 2, pp. 402-413, Apr. 2011.

[33] O. Alay, P. Liu, Y. Wang, E. Erkip, and S. S. Panwar, "Cooperative layered video multicast using randomized distributed space time codes," IEEE Trans. Multimedia, vol. 13, no. 5, pp. 1127-1140, Oct. 2011.

[34] Z. Guan, T. Melodia, and D. Yuan, "Jointly optimal rate control and relay selection for cooperative wireless video streaming," IEEE/ACM Trans. Netw., vol. 21, no. 3, pp. 1173-1186, Mar. 2013.

[35] H.-P. Shiang and M. van der Schaar, "Dynamic channel selection for multi-user video streaming over cognitive radio networks," in Proc. IEEE ICIP, San Diego, CA, USA, Oct. 2008, pp. 2316-2319.

[36] L. Ding, S. Pudlewski, T. Melodia, S. Batalama, J. Matyjas, and M. Medley, "Distributed spectrum sharing for video streaming in $\operatorname{cog}$ nitive radio ad hoc networks," in Proc. Int. Workshop Cross-Layer Des. Wireless Mobile Ad Hoc Netw., Niagara Falls, ON, Canada, Sep. 2009, pp. 1-13.

[37] H. Luo, S. Ci, and D. Wu, "A cross-layer design for the performance improvement of real-time video transmission of secondary users over cognitive radio networks," IEEE Trans. Circuits Syst. Video Technol., vol. 21, no. 8, pp. 1040-1048, Aug. 2011.

[38] Y. Ding and L. Xiao, "Video on-demand streaming in cognitive wireless mesh networks," IEEE Trans. Mobile Comput., vol. 12, no. 3, pp. 412-423, Mar. 2013.
[39] S. Katti, S. Gollakota, and D. Katabi, "Embracing wireless interference: Analog network coding," in Proc. ACM SIGCOMM, Kyoto, Japan, Aug. 2007, pp. 397-408.

[40] J. Liu, W. Chen, Z. Cao, and Y. J. Zhang, "Active interference cancellation-aided QoS-aware distributed ARQ for cognitive radios with heterogeneous traffics," EURASIP J. Wireless Commun. Netw., vol. 2013, no. 29 , Feb. 2013

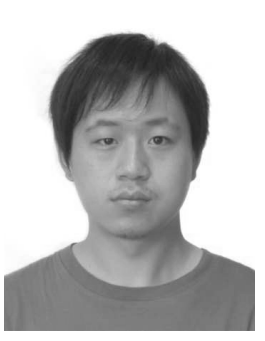

Yi Xu (S'11) received the B.S.degree in electronic information engineering from University of Electronic Science and Technology of China, Chengdu, China, and the M.S. degree in electronic engineering from Tsinghua University, Beijing, China, in 2007 and 2010, respectively. $\mathrm{He}$ is currently working toward the Ph.D. degree with the Department of Electrical and Computer Engineering, Auburn University, Auburn, AL, USA.

His research interests include optimization, cognitive radio networks. game theory, MIMO, OFDM, IDMA, and

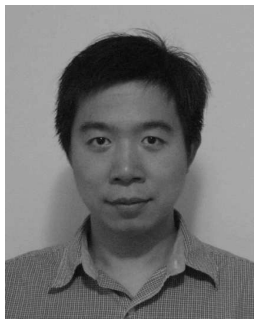

Donglin Hu received the B.S. degree in electrical engineering from Nanjing University of Posts and Telecommunications, Nanjing, China; the M.S. degree in electrical engineering from Tsinghua University, Beijing, China; and the M.S. degree in probability and statistics and the Ph.D. degree in electrical and computer engineering from Auburn University, Auburn, AL, USA, in 2004, 2007, 2011, and 2012, respectively.

He was a Post-Doctoral Fellow with Auburn University from 2012 to 2013 and is currently a Senior Member of Technical Staff with AT\&T Laboratories, San Ramon, CA, USA. His research interests include cognitive radio networks, cross-layer optimization, algorithm design for wireless network, and multimedia communications.

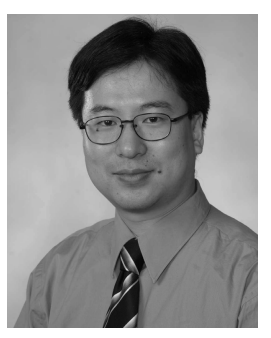

Shiwen Mao (S'99-M'04-SM'09) received the $\mathrm{Ph} . \mathrm{D}$. degree in electrical and computer engineering from Polytechnic University, Brooklyn, NY, USA, in 2004.

$\mathrm{He}$ is the McWane Associate Professor with the Department of Electrical and Computer Engineering, Auburn University, Auburn, AL, USA. His research interests include wireless networks and multimedia communications, with a focus on cognitive radio, small cells, $60-\mathrm{GHz}$ millimeter-wave networks, free space optical networks, and smart grids.

Dr. Mao is a Distinguished Lecturer of IEEE Vehicular Technology Society-Class 2014. He is on the Editorial Board of IEEE TRANSACTIONS ON WIRELESS COMMUNICATIONS, IEEE INTERNET OF THINGS Journal, IEEE COMMUNICATIONS SURVEYs AND Tutorials, Elsevier Ad Hoc Networks Journal, and Wiley International Journal on Communication Systems. He is Vice Chair-Letters (2014-2016) and the Director of the E-Letter (2012-2014) of Multimedia Communications Technical Committee (MMTC) of the IEEE Communications Society. He is a Technical Program Vice Chair for Information Systems (EDAS) of the 2015 IEEE International Conference on Computer Communications; a Symposium Co-Chair for many conferences including IEEE International Conference on Communications, IEEE Global Telecommunications, International Conference on Computer Communications and Networks, and IEEE International Conference on Industrial Technology-Southeastern Symposium on System Theory, among others; a Steering Committee Member for the IEEE International Conference on Multimedia \& Expo for 2014 to 2016 and AdhocNets, and in various roles in the organizing committees of many conferences. He received the 2013 IEEE ComSoc MMTC Outstanding Leadership Award and the NSF CAREER Award in 2010, and was co-recipient of the 2013 IEEE ICC Best Paper Award and the 2004 IEEE Communications Society Leonard G. Abraham Prize in the Field of Communications Systems. 